\title{
A viable megawatt-class space power installation under the Rankine cycle
}

\author{
R. Ferreiro Garcia \\ Department of Industrial Engineering \\ E.T.S.N.M., University of A Coruna \\ Campus of Riazor - Paseo de Ronda 51, 15011 A Coruna (Spain) \\ Fax number:+0034 981 167101, e-mail: ferreiro@udc.es;
}

\begin{abstract}
The aim of the article concerns to the description of the main achieved research results regarding the viability of a megawatt class space power plant based on the Rankine cycle. The main objectives are to find the key issues to improve the Rankine cycle efficiency. With the proposed modifications, the ideal thermal efficiency achieved using the proposed strategies increases significantly with respect to conventional power plants. This benefit associated to the reduction of the required payload capacity, reduction of the condenser radiation surface and reduction of the power plant or installation weigh; represent the main advantages of the proposed technique.
\end{abstract}

\section{Key words}

Energy conversion, Efficiency criteria, Rankine cycle efficiency; Concentrated solar power, Solar Dynamic Power System.

\section{Introduction}

In the near future, space missions such as synchronous geostationary and synchronous Low Earth Orbit (LEO) space stations will require magawat type power systems characterised by increased versatility and capabilities to deliver powers of thousands of kWe, being its power levels several times the order of magnitude greater than provided on previous and existing spacecrafts [1]. For a number of reasons, including cost, growth flexibility, and operational factors, the initial space station power generating system is shared between photo-voltaic (PV) solar cell array and solar dynamic component [2]. While the advantages of the Solar Dynamic Power System (SDPS) from its high efficiency and small size, it is also important that the technology today has been well developed for terrestrial applications. It is worth great effort to extend and perfect the SDPS technology for space applications encouraging the implementation of High-Temperature Solar Thermal (HTST) as SDPS.

SDPS can be classified according to the thermodynamic cycle as: (1) Closed Brayton Cycle(CBC), (2) Rankine Cycle(RC):alkaline metal cycle, and (3) Stirling Cycle(SC), for example, free piston Stirling engine. Detailed studies were conducted on these systems for space missions over last 50 years by NASA and other research departments [3-5].
The selected and applied technology so far, has been relayed on the CBC which has been the chosen system due to its good performance such as efficiency, which is better than RC, and due to its well-proven technology compared to free piston Stirling engine system. The CBC system operates under the efficiency of about $30 \%$, and rejects about $70 \%$ of the absorbed heat from sun radiation as waste heat to space. Based on the possibility of recovering a fraction of $\mathrm{CBC}$ waste heat, the conceptual design of cogeneration cycles combines Organic RC (ORC) or the two-phase power cycle, with CBC. A scheme of mentioned characteristics contributes increasing the global power plant efficiency, while decrease the concentrator and radiator areas, and reduce the overall power plant weight.

\section{A. The Solar Dynamic Power System (SDPS) concept}

A typically conceived SDPS includes the following major subsystems and/or components [6]:

1 High temperature solar concentrator.

2 Solar receiver with thermal energy storage devices.

3 Power conversion system, based on an advanced thermodynamic cycle.

4 Radiator to dissipate the rejected heat towards the outer space.

5 Appropriate controls and power conditioning and.

6 All the necessary auxiliary accessories required to make up the complete system.

SDPS concentrates sun-radiated heat into a receiver where the thermal energy is transferred to a heat engine for conversion into electrical power.

A power conversion system converts the heat engine's electrical output according the required applications of the spacecraft. The waste or rejected heat is removed through a heat exchanger and dissipated by radiator panels to space.

The concentrator is responsible for captures the incident solar energy and focuses it into the receiver aperture. The rigid deployed concentrator design includes a multi-panel deployable reflector with rigid reflector panels, a segmented deployment boom, and pointing and tracking mechanisms [7]. The deployment booms are inflated and then rigidized to position the reflector with respect to the focal plane [8]. 
The receiver is the key component of the SDPS converting solar energy into useful thermal energy. The most common receiver configurations are cylindrical or conical cavities with small apertures in the front plates that restrict the retro-radiation of the solar flux [9]. The working fluid is normally circulated through axial or circumferential ducts located in the receiver's circumferential walls.

A chosen material filling in the receiver is used to store thermal energy into a storage device for power production in the shade portion of a LEO allowing for continuous power delivering thus eliminating the need for electric batteries.

A block diagram of a SDPS based on an ORC is shown in figure 1 . The solar dynamic power system collects the suns rays onto a solar collector which in turn focus the solar ray into a receiver. This results in heating of the receiver which in turn heats a storage thermal fluid (STF) that heats a working fluid (WF) of a closed thermodynamic cycle to power a turbo-alternator which results in the production of electrical energy. The solar receiver transfer the thermal energy to the storage device being designed so that it transfers energy to the fluid during the on sun phase of the orbit, and stores thermal energy for operation during the shade phase. The working fluid is then cooled in the condenser associated to the radiator which rejects the waste heat to the heat sink (the outer space) [10].

\section{B. Heat Storage Media}

Various central receiver heat transfer media have been investigated, including water/steam, liquid sodium, molten salt and ambient air. Those storage systems allows solar energy to be collected during daylight hours and dispatched as high value electric power at night or when required by the utility.

For medium temperature $\left(600{ }^{\circ} \mathrm{C}\right)$ energy storage, ammonia has been used. For high temperature storage in e.g. Solar electricity power plants, molten salts (e.g. Sodium-nitrate salt) have been considered. The heat capacity of the Sodium Sulphide is 5-times the heat capacity of the water, being considered as a serious candidate. The use of energy storage in Zinc and Syngas provides temperatures approaching $1300{ }^{\circ} \mathrm{C}$

According recent published studies referred in [11], the alkali-metal RC is the primary study object of space RC system. However, the conversion efficiency of RC is lower than other cycles. For example, including alternator efficiencies, the CBC and Stirling cycles result in $35.6 \%$ and $42.0 \%$ power conversion efficiencies, respectively, but a single-stage potassium RC will result in $15 \%-20 \%$ efficiency for the range of temperatures. Addition of one or two turbine reheats raises thermodynamic efficiency to $25 \%-30 \%$, but at the expense of a two or three stage turbine and a solar receiver design capable of providing the needed lowtemperature heats [12].
The low efficiencies result in oversized concentrator, receiver, and radiator, the multi-reheat turbine and receiver complexity, and the peak operating temperature being much higher than those of other cycles. Therefore, the alkali-metal RC was eliminated from further consideration of space station [13]-[18]. Taking into account the availability of materials capable to operate under high temperatures (higher than three decades ago) while keeping good working characteristics, a lot of possibilities are present.

\section{On the improvements of the RC based technology}

According reviewed technical literature related with this topic, based on the available state of the art technology, alkali-metal based RC has been discarded as a viable mean to satisfy the power demands on space applications. Nevertheless a more efficient RC based power plant can be conceived for space needs using concentrated solar power (CSP) at relative higher temperatures into the storage device. In the actual state of the art technology, heat can be added to one side of the cycle (heat storage device) at 600 degrees to 850 degrees $C$ and rejected at the other side (the radiation based condenser) at 150 degrees to 450 degrees C. Based on such temperature ranges, opportunities to the RC and ORC are open on the basis of the proposed improvements. Proposed improvements are based on theoretical thermodynamic criteria and only technical restrictions contribute to avoid practical implementations.

\section{A. Proposed design and operating criteria}

Conventionally, the major two characteristic magnitudes that contributes to the improvement of RC efficiency, are the hot side temperature and cold side temperature as stated by Carnot efficiency definition: that means to increase the hot side temperature and decrease to cold side temperature as much as possible taking into account the inherent high and low operational limits due to safety reasons. Increasing the hot side temperature as much as possible with the inherent increase of pressure, apart the thermal efficiency increase, it means to decrease the necessary turbine or expander volume. On the other hand, reducing the cold side temperature means to increase the expander volume to let the working fluid the inherent expansion ratio.

In this contribution expansion phase is limited to a value approaching the sub-critic pressure and so the sub-critic temperature. This idea obeys to the fact that the latent heat of condensation is significantly reduced, and consequently the remaining working fluid is returning to the vaporizer with an amount of sensible heat which is being used again in the cycle as a source heat instead of being rejected towards the cold side sink heat side.

The following key issues are proposed to improve the RC efficiency:

- Increasing the specific power of the WF, meaning that increasing the WF's specific heat and temperature is an option. 
- Increase the WF temperature, which means to increase the heat concentration ratio.

- Minimisation of the rejected heat due to the condensation phase.

- Using a backpressure expander or turbine

- Minimisation of the entropy change in the condensation phase.

- Adjusting the exhaust backpressure as much as possible to achieve a value of the pressure so that the phase change or condensation is carried out under a significant lower entropy change.

According mentioned criteria, a theoretical study has been carried out to compare the behaviour of the solar dynamic power system working fluids (H2O) operating under a modified supercritical Rankine cycle.

Table 1 shows the main characteristic parametric values of the proposed SDPS operating under the modified RC for $\mathrm{H} 2 \mathrm{O}$ working fluids. Water has been considered in this preliminary research due to its special characteristics: is not expensive, high specific heat capacity and appropriate critical temperature.

Table I. - Characteristic parameters of the solar dynamic power system RC

\begin{tabular}{|l|l|}
\hline \multicolumn{1}{|c|}{ Characteristic Value of RC } & \multicolumn{1}{c|}{ H2O } \\
\hline Supercritical. temperature (K) & $800-1200$ \\
\hline Near critical exhaust temperature (K) & 647 \\
\hline Carnot efficiency (critical exhaust) T(K) & 0.44 \\
\hline Ambient temperature & 420 \\
\hline Near critical Exhaust Radiation Surface (m2) & 91 \\
\hline Sub-crit. Exhaust Radiation Surface (m2) & 2315 \\
\hline
\end{tabular}

\section{Discussion of results}

Some interesting results are achieved as consequence of a simplistic analysis:

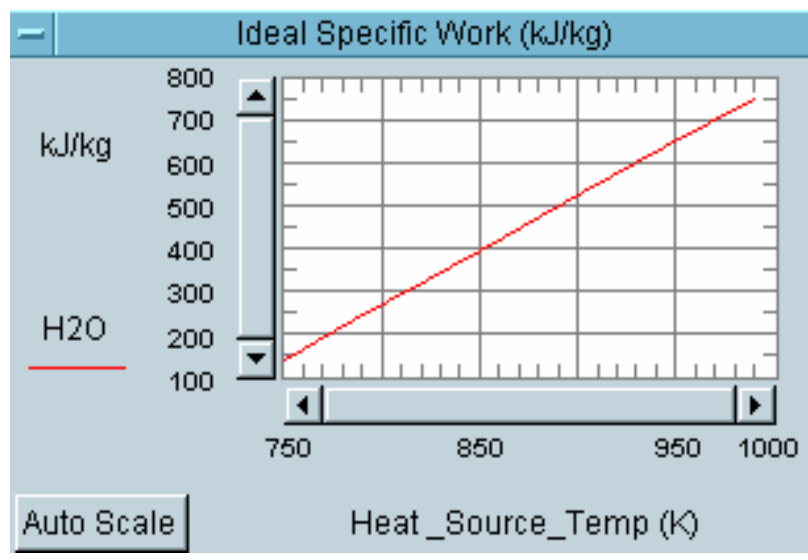

Fig. 2. The specific work $(\mathrm{kJ} / \mathrm{kg})$ as function of the hot heat source temperature $(\mathrm{K})$.

According the proposed Ranking cycle criteria, the working fluid is returning to the vaporizer with an amount of heat that is being transferred to the heat source to used again in the cycle as a recycled source heat instead of being rejected towards the cold side or sink heat side. As consequence of such contribution, the following results have been achieved:

In the figure 2, the ideal specific work is represented. As expected, the work is a direct proportional function of the heat source temperature. In the actual state of the art technology such supercritical temperature are technically viable.

In figure 3 , it is represented the ratio of the specific work to the radiation surface expressed in $(\mathrm{kJ} / \mathrm{kg}) /(\mathrm{m} 2)$. The developed work out is proportional to the condenser radiation surface.

In figure 4 it is depicted the amount of rejected heat as function of the lower condensation phase temperature. It is shown that the optimum lower condensation temperature is not the minimum temperature, but a temperature as closer as possible to the critical point of the working fluid, since the cost of the energy concerns exclusively to solar concentrator structure. This means that the amount of rejected heat and bottoming temperature are a key to improve the thermal efficiency of the Rankine cycle based on a solar concentrator.

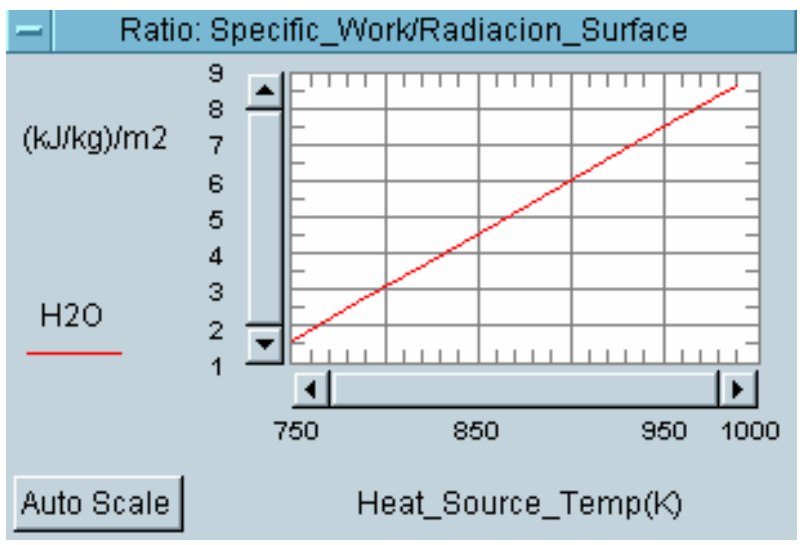

Fig. 3. The ratio of the specific work to the radiation surface $(\mathrm{kJ} / \mathrm{kg}) /$ Radiation Surface $(\mathrm{m} 2)$.

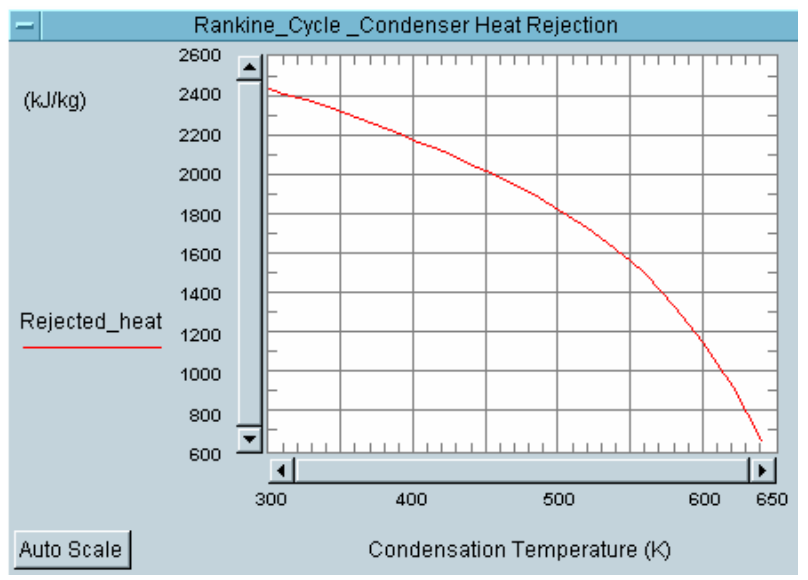

Fig. 4. The rejected heat by the condenser as function of the condensation temperature. 
Fig.5. The thermal and Carnot efficiency (\%) as function of the condensation temperature.

In figure 5 it is depicted the required condenser radiation surface as function of the environment temperature for several exhaust temperatures. It is shown that as exhaust temperatures decreases the necessary surface increases accordingly.

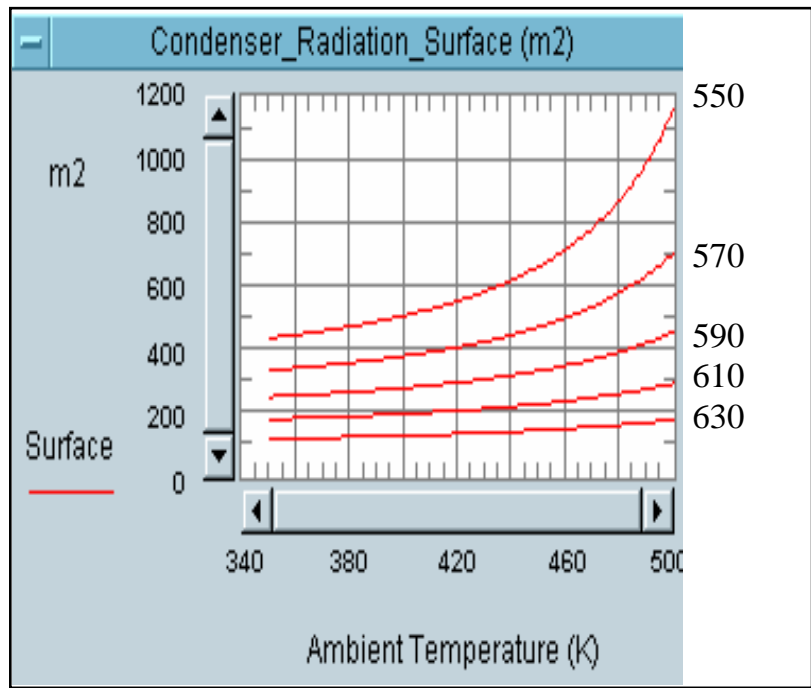

Fig.5. Condenser radiation surface area as function of ambient temperature for several exhaust or condensation temperatures.

\section{Conclusions}

Taking into account the results based on the proposed key issues with the aim of improving the RC efficiency, the ideal thermal efficiency achieved using the proposed strategies associated to the required payload capacity, condenser radiation surface and power plant or installation weight will experiment favourable dramatic changes.

The fact of modifying the Rankine cycle so that the exhaust pressure and temperature approaches the critical pressure corresponding to the applied working fluid, means that condenser radiation surface decreases drastically with a direct incidence on the installation weight.

The possibilities of application of the improved power plant in a variety of environments increases also due to the wide range of radiation temperatures of the associated with the condenser radiation.

\section{References}

[1] James P., George M., John P.. Nuclear Propulsion and Power Systems for Near Term Exploration of the Solar System. (2005), AIAA-2005-259.
[2] Roschke E J. "Solar dynamic systems for spacecraft power applications” (1986). AIAA-86-0382.

[3] Don Knasel, Derik Ehresman. "Solar Concentrator Advanced Development Program Final Report”, (1989). NASA CR-185173.

[4] Richard K S,Lee S M. "800 Hours of Operational Experiment from a 2kWe Solar Dynamic System”, (1999). NASA-TM-1999-208840..

[5] Robert P Migra. "Conceptual definition of a technology development mission for advanced solar dynamic power systems”, (1986), NASA-CR-179482.

[6] Miles O Dustin, Robert P Migra, Albert J Juhasz.”Advanced solar dynamic space power systems perspectives, requirements and technology needs", (1987). NASA-TM-88884.

[7] Don Knasel, Derik Ehresman. "Solar Concentrator Advanced Development Program Final Report”, (1989). NASA CR-185173.

[8] Frederick H. Redell, Justin Kleber, David Lichodziejewski. Inflatable-Rigidizable "Solar Concentrators for Space Power Application”, (2005). AIAA-2005-1879.

[9] Joseph N. , Kerslake, Thomas W. etc. "Modeling Cyclic Phase Change and Energy Storage in Solar Heat Receivers", (1997) AIAA-97-2452.

[10] Niggemann R, Mckenna R, Chaudoir D, et al. "A solar dynamic power conversion system for Space Station”, (1985). AIAA-85-1480, 1985.

[11] Bin Wu, Yu_ming Xing, "Space Solar Dynamic Power Systems Status and Development”, (2010). 978-1-4244-48135/10/\$25.00 (C)2010 IEEE

[12] Wayne E W and Jerry M F. Solar Dynamic Power System Definition Study, (1988). NASA-CR-180877.

[13] Takeshi HOSHINO, Hitoshi NAITO, Tsutomu FUJIHARA, et al. "Experimental study on stirling engine generator and solar receiver system for future space applications”, (2000). AIAA-2000-2842.

[14] Lanny G Thieme, Jeffy G Schreiber. „Advanced Technology Development for Stirling convertors", (2004). NASA-TM-2004-213186.

[15] Jack G Slaby. "Free-piston Stirling technology for space power”, (1989). NASA-TM-101956.

[16] Aristide M. "High-Efficiency Solar Dynamic Space Power Generation System”, (1991). ASME J. Sol Energy, 113:131145.

[17] Karl W B. "A New Power Conversion System, Heat Engine and Space Power System for Earth Orbit Applications”, (2004). AIAA 2004-5695, 2004.

[18] Thomas L.Kessler. "An overview of a solar thermal propulsion and power system demonstration applicable to HEDS”, (2001). AIAA-2001-4777. 


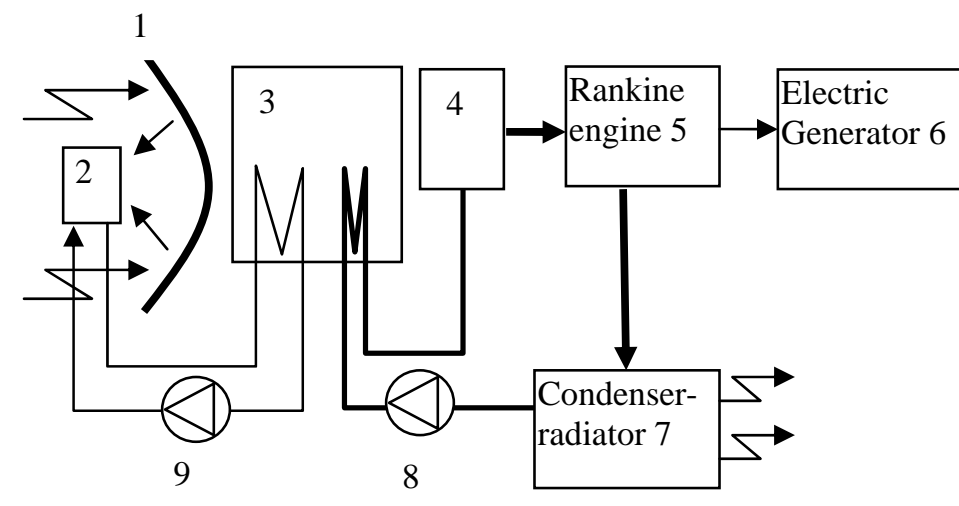

1 solar concentrator

2 heat receiver

3 heat storage media

$4 \mathrm{WF}$ vaporiser

5 turbine or expander

6 electric power generator

7 radiation based condenser

8 WF feed pump

9 STF circulation pump

Fig. 1. The general scheme of a SDPS 